\title{
Two Doses of Candidate TB Vaccine MVA85A in Antiretroviral Therapy (ART) Naïve Subjects Gives Comparable Immunogenicity to One Dose in ART+ Subjects
}

\author{
Tandakha N. Dieye ${ }^{1}$, Birahim P. NDiaye ${ }^{1}$, Alle B. Dieng ${ }^{2}$, Marema Fall ${ }^{1}$, Nathaniel Britain ${ }^{3}$, \\ Samantha Vermaak ${ }^{3}$, Makhtar Camara', Halimatou Diop-Ndiaye', Ndeye Fatou Ngom-Gueye ${ }^{4}$, \\ Papa A. Diaw ${ }^{1}$, Coumba Toure-Kane ${ }^{1}$, Papa S. Sow ${ }^{2}$, Souleymane Mboup ${ }^{1}$, Helen McShane ${ }^{3 *}$
}

1 Laboratoire de Bacteriolgie-Virologie, Centre Hospitalier Universitaire Le Dantec, Dakar, Senegal, 2 Centre de Recherche Clinique, Centre Hospitalier Universitaire de Fann, Dakar, Senegal, 3 Jenner Institute, University of Oxford, Oxford United Kingdom, 4 Centre de Traitement Ambulatoire, Centre Hospitalier Universitaire de Fann, Dakar, Senegal

\begin{abstract}
Tuberculosis (TB) is a global public health problem exacerbated by the HIV epidemic. Here we evaluate a candidate TB vaccine, MVA85A, in a Phase I study in HIV-infected adults in Senegal. 24 patients were enrolled: Group 1:12, antiretroviral therapy (ART) naïve, adults, with CD4 counts $>300$ and HIV RNA load $<100000$ copies/ml. Group 2:12 adults, stable on ART, with CD4 counts $>300$, and an undetectable HIV RNA load. Safety was evaluated by occurrence of local and systemic adverse events (AEs) and by monitoring of CD4 count, HIV RNA load, haematology and biochemistry. Immunogenicity was evaluated by ex-vivo interferon-gamma ELISpot assay. $87.7 \%$ of AEs were mild; $11.6 \%$ were moderate; and $0.7 \%$ were severe. $29.2 \%$ of AEs were systemic; $70.8 \%$ were expected local AEs. There were no vaccine-related Serious Adverse Events (SAEs) or clinically significant effects on HIV RNA load or CD4 count. In ART naive subjects, the first MVA85A immunisation induced a significant immune response at 1 and 4 weeks post-immunisation, which contracted to baseline by 12 weeks. Durability of immunogenicity in subjects on ART persisted out to 24 weeks post-vaccination. A second dose of MVA85A at 12 months enhanced immunogenicity in ART naïve subjects. Subjects on ART had higher responses after the first vaccination compared with ART naïve subjects; responses were comparable after 2 immunisations. In conclusion, MVA85A is well-tolerated and immunogenic in HIV-infected subjects in Senegal. A two dose regimen in ART naïve subjects is comparable in immunogenicity to a single dose in subjects on ART. Clinicaltrials.gov trial identifier NCT00731471.
\end{abstract}

Citation: Dieye TN, NDiaye BP, Dieng AB, Fall M, Britain N, et al. (2013) Two Doses of Candidate TB Vaccine MVA85A in Antiretroviral Therapy (ART) Naïve Subjects Gives Comparable Immunogenicity to One Dose in ART+ Subjects. PLoS ONE 8(6): e67177. doi:10.1371/journal.pone.0067177

Editor: Francesco Dieli, University of Palermo, Italy

Received February 27, 2013; Accepted May 15, 2013; Published June 28, 2013

Copyright: (c) 2013 Dieye et al. This is an open-access article distributed under the terms of the Creative Commons Attribution License, which permits unrestricted use, distribution, and reproduction in any medium, provided the original author and source are credited.

Funding: This work was supported by EuropeAID (SANTE/2006/105-066; http://ec.europa.eu/europeaid/index_en.htm), and Wellcome Trust (076943/Z/05/Z to HM; www.wellcome.ac.uk). The funders had no role in study design, data collection and analysis, decision to publish, or preparation of the manuscript.

Competing Interests: HMcS is an inventor on a patent for MVA85A and is a shareholder in a Joint Venture, the Oxford Emergent Tuberculosis Consortium, formed for the development of MVA85A. This does not alter the authors' adherence to all the PLOS ONE policies on sharing data and materials.

*E-mail: helen.mcshane@ndm.ox.ac.uk

\section{Introduction}

Tuberculosis $(\mathrm{TB})$ is one of the leading causes of death from a single infectious agent. One third of the world's population is latently infected with Mycobacterium tuberculosis $(M . t b)[1]$ and TB kills about 1.45 million people annually worldwide [2]. Latently infected individuals have a $10 \%$ lifetime risk of developing active TB disease, or a 10\% annual risk if they become immunosuppressed [3]. TB prevention remains one of today's greatest public health challenges and an efficacious vaccination strategy will be an essential tool to control it. The highest numbers of TB cases are in Africa and South East Asia. Even though TB prevalence is decreasing in all six WHO regions [2], the burden in higher incidence countries is in part due to the increasing prevalence of HIV $[4,5]$.

HIV likely serves as a driver of TB at the population level by increasing the incidence of $\mathrm{TB}$ and $\mathrm{TB}$ - related deaths in a population of immunodeficient individuals susceptible to both primary and reactivation TB [6]. The relative risk of TB doubles in the first year after HIV infection, when CD4 counts are still preserved, and continues to increase during the years after seroconversion as CD4 counts decrease [7]. In individuals with latent $M . t b$ infection, HIV accelerates and augments progression to reactivation of $\mathrm{TB}$. The initiation of $\mathrm{ART}$ can reduce the risk of HIV-associated TB by restoring the immune response to $M . t b$, leading to sustained reductions in long-term TB risk [8]. However, the initial weeks of immune recovery may be associated with a transiently heightened risk of TB [9]. Thereafter the risk of TB decreases rapidly particularly in the first $2-3$ years of ART [10]. However, even for patients on ART who have CD4 counts above 500 cells/ $\mu \mathrm{l}$, the TB incidence rate can still remain 2-fold higher than those for adults without HIV [8].

The only available vaccine, Mycobacterium bovis BCG, is largely ineffective at protecting against adult pulmonary disease, but does 
Table 1. Epidemiological and clinical characteristics of HIV-1 eligible volunteers.

\begin{tabular}{|c|c|c|}
\hline Characteristics & HIV+ART- Group $1(n=12)$ & HIV+ART+ Group $2(n=12)$ \\
\hline Age, years (Min-Max) & $35(24-44)$ & $35(30-47)$ \\
\hline \multicolumn{3}{|l|}{ Sex } \\
\hline Male & 2 & 4 \\
\hline Female & 10 & 8 \\
\hline Hemoglobin (g/dl) & $11.9(10.1-15.5)$ & $12.45(11.6-15.5)$ \\
\hline Neutrophils $\left(10^{9} / \mathrm{l}\right)$ & $1.6(1-2.3)$ & $1.8(1-2.9)$ \\
\hline Lymphocytes $\left(10^{9} / \mathrm{l}\right)$ & $1.6(1.1-2.8)$ & $1.75(1-2.6)$ \\
\hline ALAT (UI/I)* & $18(9-49)$ & $14(10-50)$ \\
\hline CD4 counts $/ \mu$ I & $572(300-1120)$ & $543(440-1281)$ \\
\hline HIV RNA load copy/ml & 3089 (40-95221) & 40 \\
\hline ART therapy & No & Yes \\
\hline M. tb infection & $7(58.3 \%)$ & $12(100 \%)$ \\
\hline
\end{tabular}

Data are median (interquartile range) values when indicated.

*ALAT: alanine transferase.

doi:10.1371/journal.pone.0067177.t001

protect against disseminated $\mathrm{TB}$ and tuberculosis meningitis in children $[11,12]$. BCG is contraindicated in HIV-infected infants, even in settings where TB is highly endemic, and a safer, more effective vaccine is urgently needed [13]. There are many new TB vaccines at different stages of development [14]. MVA85A is a candidate TB vaccine designed to enhance BCG $[15,16]$. It is a subunit-viral vectored vaccine that uses Modified Vaccinia virus Ankara as a delivery system for the mycobacterial antigen $85 \mathrm{~A}$
[17]. MVA85A boosts both antigen specific IFN- $\gamma$ secreting CD4+ and CD8+ $\mathrm{T}$ cells and can induce higher levels of protection against aerosol challenge than after BCG alone in mice, nonhuman primates and cattle [18-20]. The promising safety and immunogenicity of this candidate vaccine in UK trials has led to further Phase I and IIa clinical trials in target populations in South Africa, The Gambia and Senegal $[15,21,22]$. The immunogenicity of MVA85A has been well characterised and the immunity

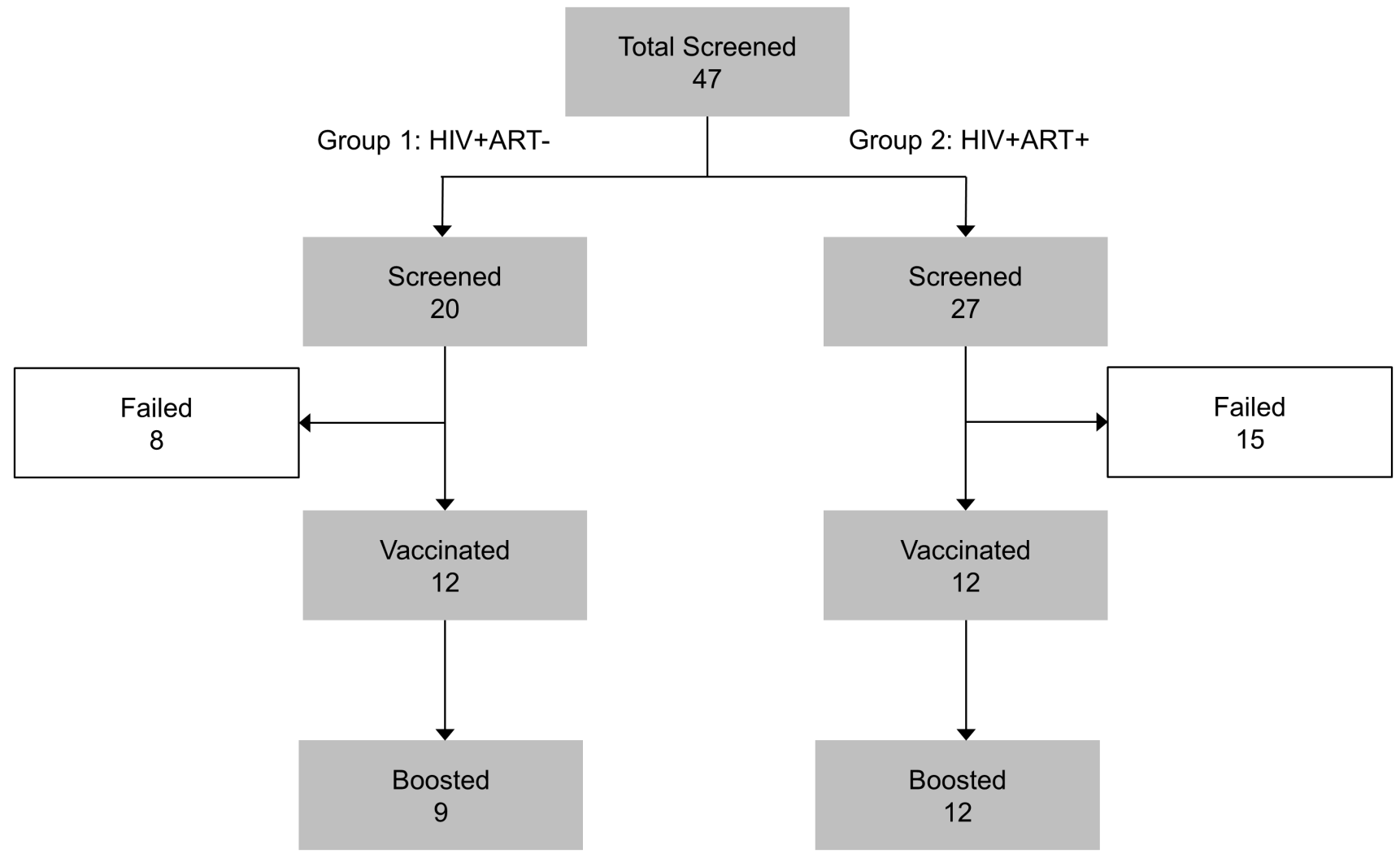

Figure 1. Consort diagram of the study population. doi:10.1371/journal.pone.0067177.g001 
induced is thought to be important for protection. A recent phase IIb safety and efficacy trial in BCG-vaccinated South African infants demonstrated MVA85A to be safe but not to enhance BCG-induced protective immunity [23]. The level of antigenspecific CD4+ T cells induced in this infant trial was modest. It is not clear whether, in populations where the immunogenicity is greater; this vaccine would confer significant protection [24]. In adults, high frequencies of antigen-specific IFN- $\gamma$-producing polyfunctional CD4+ T cells are induced by MVA85A, including expansion of a memory population, and the frequency of antigenspecific cells remains significantly higher than baseline for at least one year after vaccination [25]. HIV-infected subjects are a really important target population for a new TB vaccine and MVA85A has been safely administered to HIV-infected subjects in the UK and South Africa [26,27].

In this study, we evaluated for the first time the safety and immunogenicity of MVA85A in Senegalese HIV-infected subjects, and furthermore evaluated both comparative immunogenicity in subjects on and off anti-retroviral therapy, and whether a second, homologous boosting immunisation could improve the elicited immune response in HIV-1-infected individuals.

\section{Materials and Methods}

\section{Study Population}

24 healthy HIV-1 infected volunteers, male and female, aged 18 to 50 years were enrolled in the study after a screening visit at the Ambulatory Treatment Centre (CTA) and the Centre Régional de Recherche Clinique et de Formation at the Fann Teaching hospital between August 2008 and February 2010 (Table 1). All subjects gave written informed consent. The trial was approved by the Comité National d'Ethique pour la Recherche en Santé (GNERS) in Senegal and the Oxford Tropical Research Ethics Committee (OXTREC) in the UK. The protocol for this trial and supporting CONSORT checklist are available as supporting information (see Checklist S1 and Protocol S1).

Eligible subjects attended the Bacteriologie et Virologie Laboratoire at Le Dantec Teaching Hospital (LBV) for vaccination (Figure 1). In group 1, twelve patients were HIV-1 antibody positive; diagnosed at least 6 months previously; had CD4+ T cell counts $>300$ and CD4 count nadir 300; HIV RNA load $\leq 100,000$ copies $/ \mathrm{ml}$ and were not on ART. In group 2, twelve patients were HIV-1 antibody positive diagnosed at least 12 months previously, had CD4 counts prior to enrolment $>300$ and CD4 count nadir $>100$, were stable on the same ART regimen for at least 12 months, and had had undetectable HIV RNA loads $(<75$ copies/ml) for at least 12 months.

Exclusion criteria were focused on clinically significant abnormal findings from screening biochemistry or haematology, urinalysis, previous history of TB disease and/or treatment, any AIDS defining illness, prior receipt of a recombinant MVA85A, and chronic administration (more than 14 days) of immunosuppressive drugs. Reasons for exclusion are shown in Table 2.

HIV status was evaluated in plasma by current testing combining ELISA and Western Blotting assays. Absolute CD4+ and CD8+ cells were assayed from fresh blood samples following our routine procedure on the FACSCount ${ }^{\mathrm{TM}}$ flow cytometer (Becton Dickinson Biosciences, San Jose, CA).

The plasma HIV-1 RNA load was measured using the Abbott m2000 RealTime HIV-1 assay following manufacturer's instruction (Abbott Molecular, Des Plaines IL USA). Briefly, the Abbott RealTime HIV-1 assay is an in vitro reverse transcriptionpolymerase chain reaction (RT-PCR) assay for the quantitation of HIV-1 on the automated m2000 System or with m24sp extraction in human plasma from HIV-1 infected individuals over the range of 40 to $10,000,000$ copies $/ \mathrm{mL}$ ( $5 \log _{10}$ copies). The trial was registered on clinicaltrials.gov (number NCT00731471).

\section{Vaccination Procedures and Follow Up Visit}

All eligible subjects received two intradermal injections of $1 \times 10^{8} \mathrm{pfu}$ MVA85A, one at Day 0 and a booster vaccine approximately 6-12 months later. Eligibility was re-verified prior to the second immunisation; two subjects were excluded from boost vaccination according to the exclusion criteria and one due to pregnancy. Subjects stayed in the clinical trial unit at the Bacteriologie et Virologie Laboratoire at Le Dantec Teaching Hospital (LBV) for 60 minutes (+/ 10 mins) after vaccination for observation. After each immunisation, subjects were followed-up at weeks 1, 4, 12, and 24. At each follow-up visit, all AEs occurring in participants were reported for the clinical safety assessment. Blood was collected to assess the vaccine biological safety profile (biochemistry, haematology, CD4 count and HIV RNA load) and immunogenicity.

Table 2. Reasons for exclusion from recruitment into the trial.

\begin{tabular}{ll}
\hline & \\
\hline Total screened & Number of volunteers (\% of those screened*) \\
Enrolled & $\mathbf{4 7}(\mathbf{1 0 0})$ \\
Excluded & $\mathbf{2 4}(\mathbf{5 1 )}$ \\
Abnormal laboratory findings & $\mathbf{2 3}(\mathbf{4 9 )}$ \\
Hepatitis B or C positive & $7(15)$ \\
HIV RNA load $>100,000$ copies/ml (ART naïve group 1) & $5(11)$ \\
CD4 count $<300$ & $5(11)$ \\
Unavailable in study period & $4(9)$ \\
Previous medical history & $2(4)$ \\
Pregnant & $1(2)$ \\
Not required (recruitment full) & $1(2)$ \\
\hline
\end{tabular}

*Some subjects were excluded for more than one reason.

doi:10.1371/journal.pone.0067177.t002 

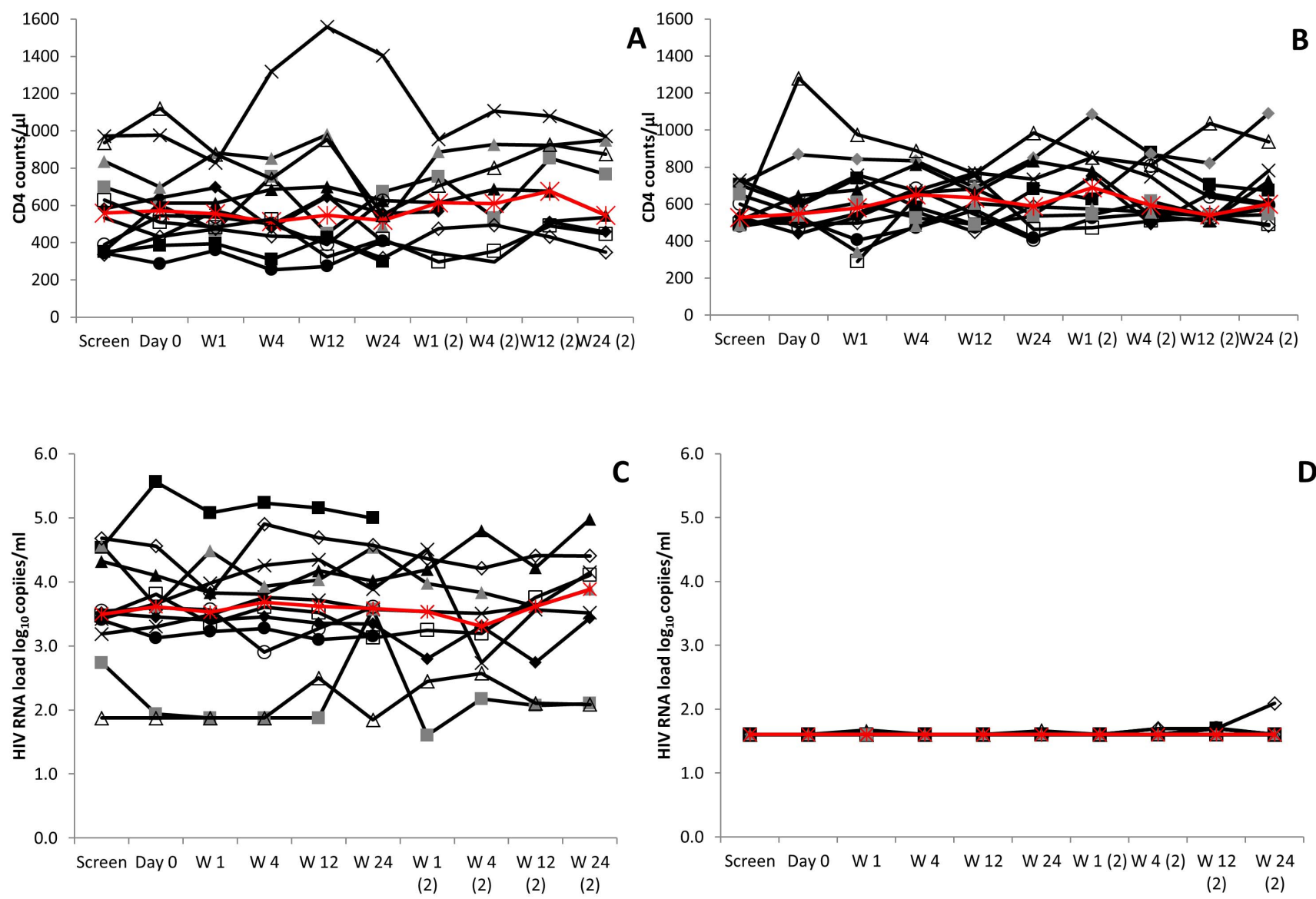

Figure 2. CD4+ T cell responses and HIV RNA load measurements after MVA85A vaccination. (A) CD4+ T cell count in 12 HIV+ART-, volunteers (group 1) and (B) in 12 HIV+ART+ volunteers (group 2). (C) HIV RNA load in 12 HIV+ART- volunteers (group 1) and (D) in 12 HIV+ART+ volunteers (group 2). Median values shown in red.

doi:10.1371/journal.pone.0067177.g002

\section{Isolation of Peripheral Blood Mononuclear Cells (PBMC)}

Briefly, 20-30 $\mathrm{ml}$ of blood was drawn into sodium heparin vacutainer tubes. The PBMC were isolated by density gradient centrifugation at $400 \times \mathrm{g}$ for 35 mins using Ficoll-Hypaque plus (Amersham, Biosciences, USA). The cells were washed in RPMI 1640 using a falcon tube to total volume of $50 \mathrm{ml}$. Cells were resuspended in $1 \mathrm{ml}$ of complete culture medium consisting of RPMI 1640 medium supplemented with 10\% FCS, 2 mM Lglutamine, $1 \mathrm{mM}$ sodium puryvate, $100 \mathrm{U} / \mathrm{ml}$ penicillin and $100 \mu \mathrm{g} / \mathrm{ml}$ streptomycin, (all Sigma Aldrich, Steinheim, Germany).

\section{ELISpot Assay}

The immunogenicity of MVA85A was determined by measuring the frequency of IFN- $\gamma$ spot-forming units (SFUs) by ex-vivo ELISpot assay using the ELISpot Kit (Mabtech, Besancon, France) as previously described [28]. ELISpot plates (Millipore, Tullagreen, Carrigtwohill, Ireland) were coated with $50 \mu \mathrm{l}$ of anti IFN- $\gamma \mathrm{Ab}$ at a final concentration of $15 \mu \mathrm{g} / \mathrm{ml}$ in the carbonatebicarbonate buffer $0.5 \mathrm{M}$. After overnight incubation, plates were washed 5 times in sterile phosphate buffered saline (PBS) and blocked by adding $100 \mu \mathrm{l}$ of complete culture medium to each well and incubating for $2-5 \mathrm{~h}$ at $37^{\circ} \mathrm{C}$. After discarding the blocking solution the isolated PBMCs, in a concentration of $0.3 \mathrm{x}$ $10^{6} \mathrm{PBMC} / 100 \mu \mathrm{l}$ were added to duplicate wells and incubated in the presence of the antigen or controls. Recombinant antigen
85A (Lionex) was used at a final concentration of $10 \mu \mathrm{g} / \mathrm{ml}$. Peptides (Peptide Protein Research, UK) were used as a single peptide pool of 6615 mers which spanned the length of antigen 85A (overlapping by 10 amino acids; at $2 \mu \mathrm{g} / \mathrm{ml}$ each), and as 7 summed pools of 9-10 overlapping 15 mer peptides. M. $t b$ PPD (purified protein derivative; $10 \mu \mathrm{g} / \mathrm{ml}$; SSI) was also used as a recall antigen. ESAT-6/CFP10 (Peptide Protein Research, UK) peptides pools were added at the final concentration of $10 \mu \mathrm{g} / \mathrm{ml}$ to detect latent $M$. $t b$ infection. Medium alone served as a negative control, and phytohemagglutinin $(10 \mu \mathrm{g} / \mathrm{ml}$; Sigma $)$ was the positive control.

ELISpot plates were incubated for $18-20$ hours at $37^{\circ} \mathrm{C}$ and then washed 5 times with PBS/0.05\% Tween20. $50 \mu \mathrm{l}$ of diluted biotin anti-IFN- $\gamma$ AB 1/1000 in PBS was added and incubated for 1 hour and then washed 5 times with PBS 0.05\% Tween 20. Finally, $50 \mu \mathrm{l}$ of developing buffer (BIO-RAD, UK) was added to each well and incubated 5-10 minutes at room temperature until distinct spots developed. ELISpot plates were washed 3 times in tap water, dried overnight and read using the AID ELISpot reader (Strassberg, Germany).

The number of IFN- $\gamma$-secreting cells (ISC) was standardised per $10^{6}$ PBMC. The response to each epitope or antigen was considered positive if the number of ISC was i) greater than twice the response without antigen stimulation, after deduction of the background level with unstimulated cells, and ii) at least 5 spots more than the negative control well, and iii) two negative control 
Table 3. IFN- $\gamma$ secreting cells (ISC) with the sum of peptides (A), the single pool (B) per $10^{6}$ PBMCs to the HIV+ART- group and $\mathrm{HIV}+\mathrm{ART}+$ group at the baseline timeline versus post vaccination.

\begin{tabular}{|c|c|c|c|c|c|}
\hline \multirow[t]{2}{*}{ A } & & \multicolumn{4}{|c|}{ Summed peptide pools } \\
\hline & & Day 0 vs W1 & Day 0 vs W4 & Day 0 vs W12 & Day 0 vs W24 \\
\hline \multirow[t]{2}{*}{ HIV+ART- Group (first dose) } & Median & 31 vs 901 & 31 vs202 & 31 vs 108 & 31 vs 84 \\
\hline & $P^{*}$ & $(<0.0003)$ & $(<0.05)$ & $(<0.04)$ & $(0.2)$ \\
\hline \multirow[t]{2}{*}{ HIV+ART- Group (second dose) } & Median & 31 vs 1517 & 31 vs 4358 & 31 vs 526 & 31 vs 212 \\
\hline & $P^{*}$ & $(<0.0001)$ & $(<0.0001)$ & $(<0.0001)$ & $(<0.0008)$ \\
\hline \multirow[t]{2}{*}{ HIV+ART+ Group (first dose) } & Median & 446 vs 3146 & 446 vs 2693 & 446 vs 1494 & 446 vs 997 \\
\hline & $P^{*}$ & $(<0.0001)$ & $(<0.0003)$ & $(<0.01)$ & $(<0.03)$ \\
\hline \multirow[t]{2}{*}{ HIV+ART+ Group (second dose) } & Median & 446 vs 3339 & 446 vs 2662 & 446 vs 1444 & 446 vs 1131 \\
\hline & $P^{*}$ & $(<0.002)$ & $(<0.0001)$ & $(<0.003)$ & $(<0.0007)$ \\
\hline \multirow[t]{2}{*}{ B } & & \multicolumn{4}{|c|}{ Single pool peptides } \\
\hline & & Day 0 vs W1 & Day 0 vs W4 & Day 0 vs W12 & Day 0 vs W24 \\
\hline \multirow[t]{2}{*}{ HIV+ART- Group (first dose) } & Median & 8 vs 423 & 8 vs 50 & 8 vs 21 & 8 vs 23 \\
\hline & $P^{*}$ & $(<0.0001)$ & $(<0.06)$ & (0.1) & $(0.1)$ \\
\hline \multirow[t]{2}{*}{ HIV+ART- Group (second dose) } & Median & 8 vs 478 & 8 vs 597 & 8 vs 140 & 8 vs 133 \\
\hline & $P^{*}$ & $(<0.0001)$ & $(<0.0001)$ & $(<0.001)$ & $(<0.007)$ \\
\hline \multirow[t]{2}{*}{ HIV+ART+ Group (first dose) } & Median & 68 vs 580 & 68 vs 317 & 68 vs 233 & 68 vs 181 \\
\hline & $P^{*}$ & $(<0.0004)$ & $(<0.0002)$ & $(<0.01)$ & $(<0.05)$ \\
\hline \multirow[t]{2}{*}{ HIV+ART+ Group (second dose) } & Median & 68 vs 389 & $68 \vee 428$ & 68 vs 196 & 68 vs 220 \\
\hline & $P^{*}$ & $(<0.0009)$ & $(<0.002)$ & $(<0.01)$ & $(<0.004)$ \\
\hline
\end{tabular}

*Mann Whitney test.

doi:10.1371/journal.pone.0067177.t003

wells containing only complete medium and PBMC had less than 20 spots per well, and $i v)$ if the counts were $>15$ spots/well, the count between duplicates could not vary by more than $50 \%$ in a total of $90 \%$ of all wells.

\section{Statistical Analysis}

The data were analysed using the GraphPad Prism software version 5 statistical package (Software MacKiev, GraphPad, San Diego, CA). For non-normally distributed data the Mann-Whitney $U$ test was used to compare two groups and $P<0.05$ was considered significant. All tests of significance were two tailed. Comparison of $M . t b$ infection rates between the two groups was done using a Fisher's exact test.

\section{Results}

\section{Characteristics of the Study Population}

All study groups were similar in parameters shown on Table 1 at screening, except for the HIV RNA load and antiretroviral treatment (ART). Hepatitis markers, HBS antigen, anti$\mathrm{HB}$ core and hepatitis $\mathrm{C}$ antibodies were negative for all HIV-1 infected volunteers.

Using the ESAT/CFP10 ex-vivo ELISpot, 58.3\% (7/12) of HIV+ART- volunteers (group 1) were latently infected with $M . t b$ and all HIV+ART+ volunteers (group 2) were identified as $M . t b$ infected $(p=0.0373)$.

\section{MVA85A Clinical Safety Data}

Most of the AEs $(87.7 \%$; 264/301) were mild in severity; $11.6 \%$ $(35 / 301)$ were moderate and the remaining $0.7 \%$ (2/301) were severe. Systemic AEs made up 29.2\% (88/301) of the total, and symptoms included headaches, fever, fatigue, and/or joint pain, all of which spontaneously resolved. 70.8\% (213/301) of AEs were expected local AEs. There were no vaccine-related SAEs observed during the study.

\section{MVA85A Biological Safety Data}

Hematology and Biochemistry parameters. Only one HIV+ART- volunteer had a transient neutropenia at week 1 post booster dose, where the neutrophil count dropped from $1.5 \mathrm{x}$ $10 * 9 / 1$ to $0.6 \times 10 * 9 / 1$ and recovered at 12 weeks. This was not considered clinically significant and the patient remained asymptomatic throughout.

GD4 counts and HIV RNA load monitoring. CD4 counts remained stable during the study (Figure 2A\&B). There were no clinically significant changes in CD4 count throughout this study.

Median CD4 count/ $\mu$ l was similar in the HIV+ART- group at day 0 at the first (572) and at the booster dose (658); $\mathrm{p}=0.4$. Similar CD4 counts were observed in the HIV+ART+ group at day 0 at the first vaccination (543) and at the booster dose (587); $\mathrm{p}=0.7$.

No clinically significant changes in HIV RNA load were seen in any of the 24 subjects (Figure 2G, 2D). All HIV+ART+ volunteers had undetectable HIV RNA load levels except one individual who had a detectable HIV RNA load (123 copies/ml) at week 24 post second vaccination. This was not considered clinically significant.

MVA85A immunogenicityMVA85A-specific IFN- $\gamma$ producing $T$ cell responses in the HIV+ART- group. A significant increase in Ag85A CD4+ T cells to peptides (pooled 


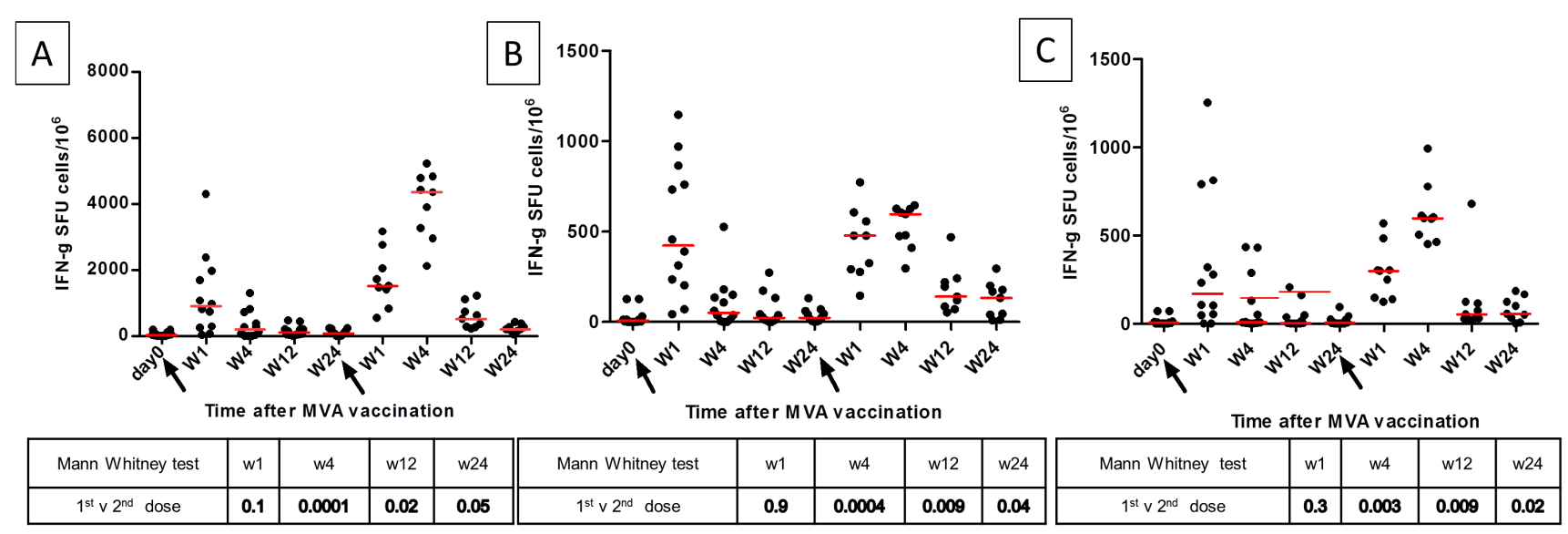

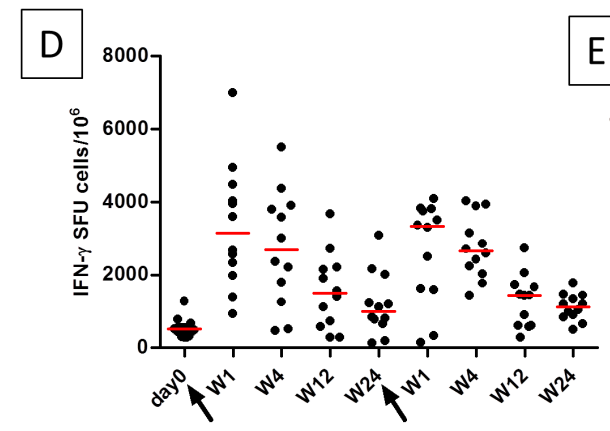

Time after MVA vaccination

\begin{tabular}{|c|c|c|c|c|}
\hline Mann Whitney test & $w 1$ & $w 4$ & $w 12$ & $w 24$ \\
\hline $1^{\text {st }} v 2^{\text {nd }}$ dose & $\mathbf{0 . 8}$ & $\mathbf{0 . 8}$ & $\mathbf{0 . 7}$ & $\mathbf{0 . 7}$ \\
\hline
\end{tabular}

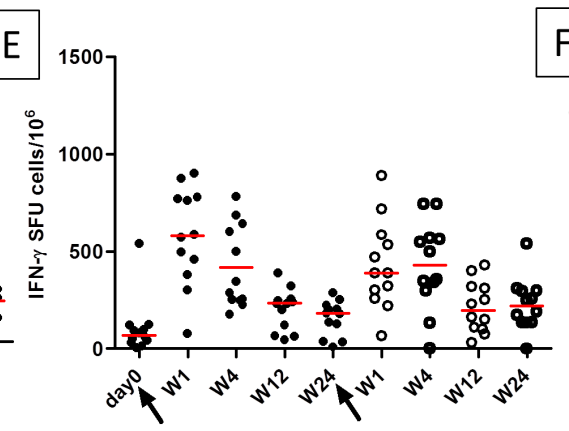

Time after MVA vaccination

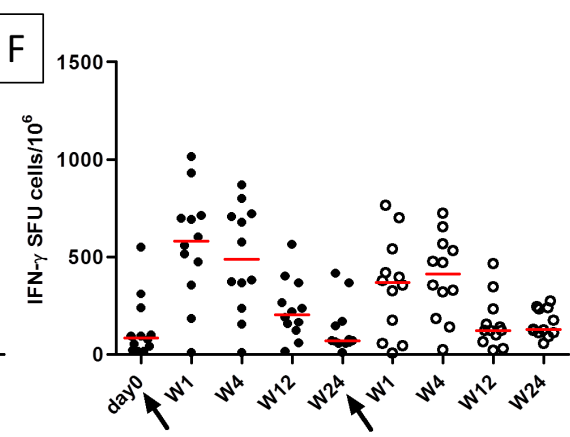

\begin{tabular}{|c|c|c|c|c|}
\hline Mann-Whitney test & w1 & w4 & w12 & w24 \\
\hline $1^{\text {st }} \vee 2^{\text {nd }}$ dose & $\mathbf{0 . 1}$ & $\mathbf{0 . 6}$ & $\mathbf{0 . 8}$ & $\mathbf{0 . 2}$ \\
\hline
\end{tabular}

Figure 3. Immunogenicity of MVA85A - HIV+ART- group (A, B, C) compared to HIV+ART+ group (D, E, F). Measured by ex vivo ELISpot assay in the sum of pool of antigen $85 \mathrm{~A}$ peptides (A, D), the single pool of 66 peptides antigen $85 \mathrm{~A}(\mathbf{B}, \mathbf{E})$ and in the recombinant antigen $85 \mathrm{~A}(\mathbf{C}, \mathbf{F})$. The figures also compare the responses after the first (arrow) and second vaccination (arrow).

doi:10.1371/journal.pone.0067177.g003

and single) and recombinant antigen was found after the first vaccination in the HIV+ART- group. At 4 weeks this response was still higher than baseline $(p=0.04)$. At 12 weeks post vaccination, only the summed pooled peptide response remained higher than baseline $(\mathrm{p}=0.046)$. At six months the summed peptide pool response was not significantly above baseline ( $p=0.26)$ (Table 3).

After the second vaccination the HIV+ART- group had a significant increase in Ag85A CD4+ T cell response to peptides (pooled and single) and recombinant antigen. At week 24 this response remained significantly above baseline (recombinant antigen, $\mathrm{p}=0.014$; single peptide pool $\mathrm{p}=0.0075$; summed peptide pool $\mathrm{p}=0.0008$ ) (Table 3).

When we compared the magnitude of IFN- $\gamma$ producing $\mathrm{T}$ cell responses after the first and second vaccination, the response was not significantly different at 1 week with the sum of pooled peptide, the single 66 peptides, or the recombinant $85 \mathrm{~A}$ antigen $(\mathrm{p}=0.30 ; 0.80$ and 0.35 respectively) (Figure 3A, 3B, 3C). However at week 4, the response after the second dose was significantly higher than after the first with the sum of pooled peptides $(p=0.0039)$, the single 66 peptides $(p=0.0039)$ and the recombinant $85 \mathrm{~A}$ antigen $(\mathrm{p}=0.0039)$ (Figure 3A, 3B, 3G). At 12 weeks, the response remained significantly higher after the second dose when compared to the first for the sum of total peptides $(p=0.023)$ and the single pooled peptides $(p=0.043)$ (Figure 3A, 3B) but not with recombinant antigen $(p=0.20)$. At
24 weeks post vaccination, the response remained higher after the second vaccination compared to the first for summed peptide pools $(p=0.030)$ the single peptide pool $(p=0.027)$ and with recombinant antigen $(p=0.0078)$ (Figure 3A, 3B, 3G).

MVA85A-specific IFN- $\gamma$-producing $T$ cell responses in the HIV+ART+ group. Baseline responses in the HIV+ART+ group were higher than in the HIV+ART- group for all antigens (summed peptide pool $\mathrm{p}=0.0001$; single peptide pool $\mathrm{p}=0.01$; recombinant antigen $\mathrm{p}=0.0004)$. In the HIV+ART+ group, there was a significant increase in Ag85A CD4+ $\mathrm{T}$ cell response to peptides (pooled and single) and recombinant antigen after the first and second vaccination. At 24 weeks this response remained higher than baseline after both first and second vaccination. IFN$\gamma$-producing $\mathrm{T}$ cell responses were significantly higher than baseline at week 1, 4, 12 and 24 but no variation was found when we compared the first vaccination to the second vaccination. There was no difference in magnitude of responses after the first and second immunisation in HIV+ART+ volunteers (Figures 3D-F).

ELISpot responses of HIV+ART- versus HIV+ART+ groups after the first vaccination. The baseline pre-vaccination responses to $\mathrm{PPD}$, recombinant $85 \mathrm{~A}$ and the peptide pools were higher in the HIV+ART+ group than in the HIV+ARTgroup (Table 4A, 4B). After vaccination, immune responses to all antigens were significantly higher in the HIV+ART+ group than in the HIV+ART- group for all time points except week 1 post- 
Table 4. IFN- $\gamma$ secreting cells (ISC) with the summed peptide pools (A, C), the single pool (B, D) per $10^{6}$ PBMCs for the HIV+ARTand HIV+ART+ groups after the first dose and second dose of MVA85A.

\begin{tabular}{|c|c|c|c|c|c|c|c|}
\hline \multirow{2}{*}{\multicolumn{2}{|c|}{$\begin{array}{l}\text { A } \\
\text { Summed peptide pools }\end{array}$}} & \multicolumn{6}{|l|}{$1^{\text {st }}$ Dose } \\
\hline & & \multirow{2}{*}{$\begin{array}{l}\text { Day } 0 \\
31\end{array}$} & W1 & \multicolumn{2}{|l|}{ W4 } & W12 & \multirow{2}{*}{$\begin{array}{l}\text { W24 } \\
84\end{array}$} \\
\hline HIV+ART- Group & Median & & 901 & 202 & & 108 & \\
\hline & (Min-Max) & $(0-195)$ & $(46-4298)$ & $(10-1305)$ & & $(9-476)$ & $(7-244)$ \\
\hline \multirow[t]{2}{*}{ HIV+ART+ Group } & Median & 446 & 3142 & 2693 & & 1494 & 997 \\
\hline & (Min-Max) & $(289-1281)$ & (948-6996) & $(478-550$ & & $(290-3677)$ & $(140-3081)$ \\
\hline$P^{*}$ & & 0.0001 & 0.002 & 0.0002 & & 0.0001 & 0.0003 \\
\hline B & & \multicolumn{6}{|l|}{$1^{\text {st }}$ Dose } \\
\hline Single pool peptides & & Day 0 & w1 & \multicolumn{2}{|l|}{ W4 } & W12 & W24 \\
\hline \multirow[t]{2}{*}{ HIV+ART- Group } & Median & 8 & 423 & \multicolumn{2}{|l|}{50} & 21 & 23 \\
\hline & (Min-Max) & $(0-127)$ & $(43-1147)$ & \multicolumn{2}{|l|}{$(0-527)$} & $(0-273)$ & $(0-132)$ \\
\hline \multirow[t]{2}{*}{ HIV+ART+ Group } & Median & 68 & 580 & \multicolumn{2}{|l|}{317} & 233 & 181 \\
\hline & (Min-Max) & $(4-539)$ & $(78-900)$ & \multicolumn{2}{|c|}{$(176-782)$} & $(47-389)$ & $(9-288)$ \\
\hline \multicolumn{2}{|l|}{$P^{*}$} & 0.01 & 0.4 & \multicolumn{2}{|l|}{0.003} & \multirow[t]{2}{*}{0.002} & 0.003 \\
\hline \multicolumn{2}{|l|}{ C } & $2^{\text {nd }}$ Dose & & & & & \\
\hline Sum pool peptides & & W1 & W4 & & W12 & & W24 \\
\hline HIV+ART - Group & Median & 1517 & 4358 & & 526 & & 212 \\
\hline & (Min-Max) & $(547-3172)$ & $(2125-5225)$ & & $(225-1220)$ & & $(57-431)$ \\
\hline HIV+ART+ Group & Median & 3339 & 2662 & & 1444 & & 1131 \\
\hline & (Min-Max) & $(159-4096)$ & (1440-4033) & & $(296-2746)$ & & $(510-1793)$ \\
\hline$P^{*}$ & & 0.07 & 0.01 & & 0.01 & & 0.0001 \\
\hline D & & $2^{\text {nd }}$ Dose & & & & & \\
\hline Single pool peptides & & W1 & W4 & & W12 & & W24 \\
\hline HIV+ART- Group & Median & 478 & 597 & & 140 & & 133 \\
\hline & (Min-Max) & $(145-773)$ & $(297-646)$ & & $(53-469)$ & & (9-296) \\
\hline HIV+ART+ Group 2 & Median & 389 & 428 & & 196 & & 220 \\
\hline & (Min-Max) & $(67-889)$ & $(3-745)$ & & $(30-429)$ & & $(0-539)$ \\
\hline$P^{*}$ & & 0.5 & 0.3 & & 0.5 & & 0.1 \\
\hline
\end{tabular}

Mann Whitney test was used.

doi:10.1371/journal.pone.0067177.t004

vaccination where the variance is higher with the single pool $(\mathrm{p}=0.4)(\mathbf{T a b l e} \mathbf{4 A}, \mathbf{4 B})$.

ELISpot responses of HIV+ART - versus HIV+ART+ groups after the second vaccination. Post second vaccination, immune responses to the summed peptide pools were significantly higher in the HIV+ART+ group compared to the HIV+ART - group at week 12 and week 24 (Table 4G), but immune responses to the recombinant antigen and single peptide pools were comparable (Table 4C, 4D).

ELISpot responses of HIV+ART - group after second vaccination versus HIV+ART+ group after the first vaccination. Comparing immune responses after the second vaccination in the HIV+ART - group to the first vaccination in the HIV+ART+ group, post vaccination responses to summed peptide pools were higher in the HIV+ART+ group than in the HIV+ART - group (Table 5A). Responses to the single peptide pool antigen were comparable between groups (Table 5B).

\section{Discussion}

MVA85A was found to be well tolerated in HIV-infected subjects in Senegal, consistent with findings from a UK study in HIV-infected subjects [26]. AEs were consistent with previously published trials $[26,29]$. No clinically significant effects on either HIV RNA load or CD4 count were observed in this study.

The magnitude of the antigen $85 \mathrm{~A}$-specific immune response was high one week post vaccination, but contracted quickly and was no longer above baseline 4 weeks after vaccination in the ART naïve HIV+ART - group 1. A previous study using MVA85A in HIV negative adult volunteers in The Gambia show a better frequency in IFN- $\gamma$ specific response against $85 \mathrm{~A}$ peptides and this amplitude was maintained after 12 weeks [21]. This strong immune response in a genetically and mycobacteriologically similar population suggests that the reduced durability seen in this study is related to chronic HIV- infection. Others have shown that CD4+ T cells from HIV infected individuals failed to secrete IFN- $\gamma$ by ex vivo ELISpot after stimulation with Hepatitis B, Hepatitis A and tetanus toxoid [30]. These naive CD4+ T cells are the subset most reduced in chronic HIV infection, and this frequently results in a loss of CD4 cellular and humoral specific immune response to HIV, pathogens and vaccines [31-33].

Importantly, the administration of a second dose of MVA85A in HIV-1 positive individuals, 12 months after the first immunisation, resulted in a significantly stronger effector response that was maintained out to 24 weeks post vaccination. This suggests that a 
Table 5. IFN- $\gamma$ secreting cells (ISC) with the summed peptide pools (A), the single pool (B) per $10^{6}$ PBMCs for the HIV+ART - group (second dose) versus the HIV+ART+ group (first dose).

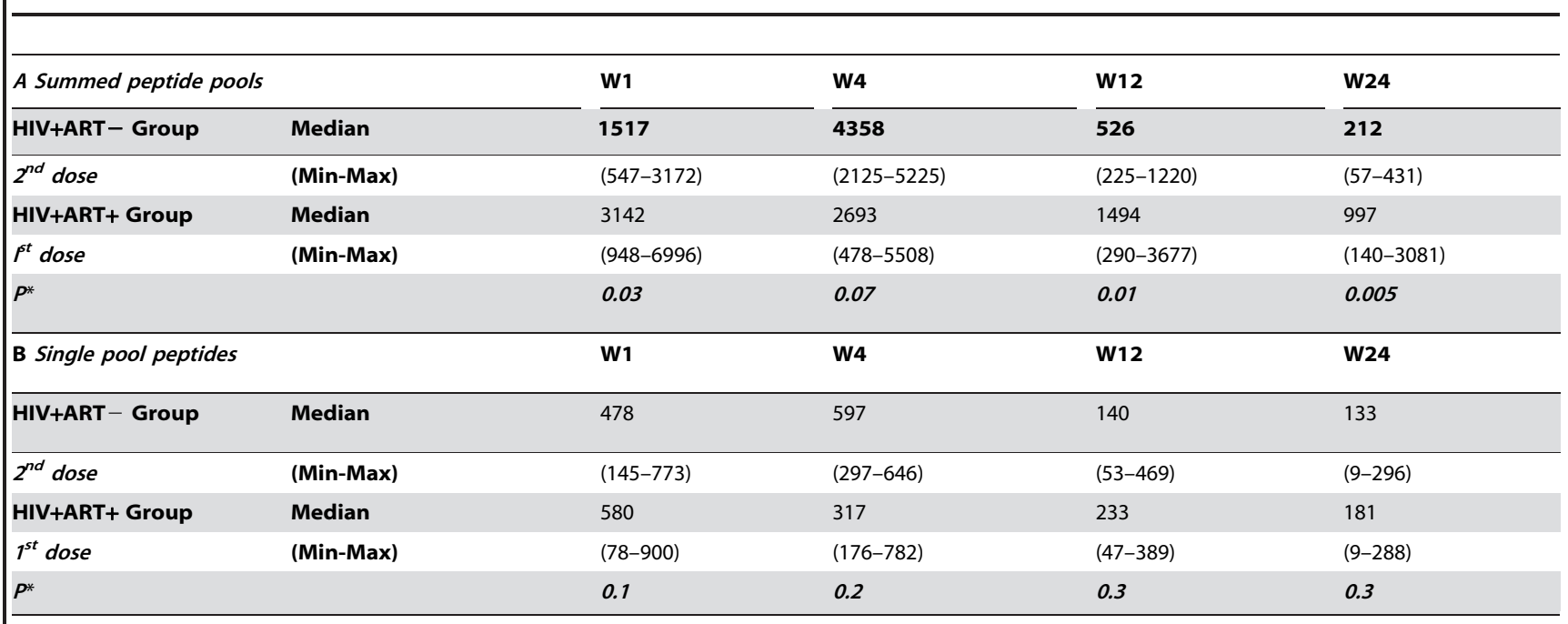

*Mann Whitney test.

doi:10.1371/journal.pone.0067177.t005

booster dose may be necessary in ART naïve, HIV infected people. Although the proportion of $M$. $t b$ infected subjects differed between the two groups, previous studies have shown no difference in T cell immunogenicity after MVA85A when comparing BCG vaccinated and $M . t b$ infected subjects [27,34]. Current standard of care in Senegal is to commence ARTs when a patient's CD4+ T cell count is less than 350 , in line with guidelines throughout the rest of the world.

ART reduces the incidence of $\mathrm{TB}$ disease in $\mathrm{HIV}$-infected adults by up to $90 \%$ [35]. TB is the predominant disease associated with immune reconstitution inflammatory syndrome (IRIS) seen during the early stage of ART [8]. This phenomenon has been estimated to account for approximately $40 \%$ of TB cases presenting during early ART, however, the risk decreases rapidly in the first 2-3 years of ART [10]. Based on the important role of Thl cells in TB, it may be more effective to vaccinate healthy HIV-1 individuals who have their immune response reconstituted by ART. Increased levels of naive GD4 counts in blood correlate to total CD4 cells after starting ART because cells are preferentially depleted in lymphoid tissues during HIV infection $[36,37]$. In this study, the immune responses post vaccination with MVA85A in subjects stable on ART were significantly higher than those seen in subjects not on ART, despite controlling for CD4 count. Furthermore, a single dose of MVA85A in subjects on ART induced a comparable response to two immunisations in ART naive subjects. There is considerable evidence to suggest that IFN$\gamma$ is necessary for protective immunity against $M$. $t b$, but it may not be sufficient [38-40]. Limited blood volumes in this study meant it was not possible to evaluate in more detail the cellular immune

\section{References}

1. Dye C, Scheele S, Dolin P, Pathania V, Raviglione MC (1999) Consensus statement. Global burden of tuberculosis: estimated incidence, prevalence, and mortality by country. WHO Global Surveillance and Monitoring Project. Jama 282: 677-686.

2. (2011) Global tuberculosis control: WHO report 2011.

3. Corbett EL, Watt CJ, Walker N, Maher D, Williams BG, et al. (2003) The growing burden of tuberculosis: global trends and interactions with the HIV epidemic. Arch Intern Med 163: 1009-1021.

4. de Colombani P, Banatvala N, Zaleskis R, Maher D (2004) European framework to decrease the burden of TB/HIV. Eur Respir J 24: 493-501. response induced by vaccination. Further studies are needed to evaluate the effects of 1 and 2 immunisations on other aspects of cellular immunity, including the more functional mycobacterial growth inhibition assays [41]. Furthermore, given the promising safety and immunogenicity data from this study, evaluation of the protective efficacy of this vaccine in HIV- infected subjects is merited. A phase IIb proof-of-concept efficacy trial is ongoing in HIV-infected subjects in South Africa and Senegal which will address this issue (clinicaltrials.gov identifier NCT01151189).

\section{Supporting Information}

Ghecklist S1 GONSORT Checklist. (DOG)

Protocol S1 Trial Protocol. (PDF)

\section{Acknowledgments}

The authors would like to thank all volunteers who took part in this trial. We thank Alison Lawrie for help with the IMP management.

\section{Author Contributions}

Conceived and designed the experiments: TND BPND ABD NB SM HM. Performed the experiments: TND BPND ABD MF MC HDN NFNG PAD CTK PSS. Analyzed the data: TND BPND ABD SV HM. Wrote the paper: TND BPND ABD SV HM. Reviewed and commented on draft: TND BPND ABD MF NB SV MC HDN NFNG PAD CTK PSS SM HM.

5. Corbett EL, Marston B, Churchyard GJ, De Cock KM (2006) Tuberculosis in sub-Saharan Africa: opportunities, challenges, and change in the era of antiretroviral treatment. Lancet 367: 926-937.

6. Bekker L-G, Wood R (2010) The changing natural history of tuberculosis and HIV coinfection in an urban area of hyperendemicity. Clin Infect Dis 50 Suppl 3: 208-214.

7. Sonnenberg P, Glynn JR, Fielding K, Murray J, Godfrey-Faussett P, et al. (2005) How soon after infection with HIV does the risk of tuberculosis start to increase? A retrospective cohort study in South African gold miners. J Infect Dis 191: 150158. 
8. Lawn SD, Myer L, Edwards D, Bekker L-G, Wood R (2009) Short-term and long-term risk of tuberculosis associated with CD4 cell recovery during antiretroviral therapy in South Africa. AIDS 23: 1717-1725.

9. Breen RAM, Smith CJ, Cropley I, Johnson MA, Lipman MCI (2005) Does immune reconstitution syndrome promote active tuberculosis in patients receiving highly active antiretroviral therapy? AIDS 19: 1201-1206.

10. Lawn SD, Badri M, Wood R (2005) Tuberculosis among HIV-infected patients receiving HAART: long term incidence and risk factors in a South African cohort. AIDS 19: 2109-2116.

11. Rodrigues LC, Diwan VK, Wheeler JG (1993) Protective effect of BCG against tuberculous meningitis and miliary tuberculosis: a meta-analysis. Int J Epidemiol 22: $1154-1158$.

12. Trunz BB, Fine P, Dye C (2006) Effect of BCG vaccination on childhood tuberculous meningitis and miliary tuberculosis worldwide: a meta-analysis and assessment of cost-effectiveness. Lancet 367: 1173-1180.

13. Hesseling AC, Caldwell J, Cotton MF, Eley BS, Jaspan HB, et al. (2009) BCG vaccination in South African HIV-exposed infants-risks and benefits. S Afr Med J 99: 88-91.

14. Kaufmann SHE, Hussey G, Lambert P-H (2010) New vaccines for tuberculosis. Lancet 375: 2110-2119.

15. Hawkridge T, Scriba TJ, Gelderbloem S, Smit E, Tameris M, et al. (2008) Safety and immunogenicity of a new tuberculosis vaccine, MVA85A, in healthy adults in South Africa. J Infect Dis 198: 544-552.

16. McShane H, Pathan AA, Sander CR, Keating SM, Gilbert SC, et al. (2004) Recombinant modified vaccinia virus Ankara expressing antigen $85 \mathrm{~A}$ boosts BCG-primed and naturally acquired antimycobacterial immunity in humans. Nat Med 10: 1240-1244.

17. McShane H, Brookes R, Gilbert SC, Hill AV (2001) Enhanced immunogenicity of CD4(+) t-cell responses and protective efficacy of a DNA-modified vaccinia virus Ankara prime-boost vaccination regimen for murine tuberculosis. Infect Immun 69: 681-686.

18. Goonetilleke NP, McShane H, Hannan CM, Anderson RJ, Brookes RH, et al. (2003) Enhanced immunogenicity and protective efficacy against Mycobacterium tuberculosis of bacille Calmette-Guerin vaccine using mucosal administration and boosting with a recombinant modified vaccinia virus Ankara. J Immunol 171: 1602-1609.

19. Verreck FA, Vervenne RA, Kondova I, van Kralingen KW, Remarque EJ, et al. (2009) MVA.85A boosting of BCG and an attenuated, phoP deficient M. tuberculosis vaccine both show protective efficacy against tuberculosis in rhesus macaques. PLoS ONE 4: e5264.

20. Vordermeier HM, Villarreal-Ramos B, Cockle PJ, McAulay M, Rhodes SG, et al. (2009) Viral booster vaccines improve Mycobacterium bovis BCG-induced protection against bovine tuberculosis. Infect Immun 77: 3364-3373.

21. Brookes RH, Hill PC, Owiafe PK, Ibanga HB, Jeffries DJ, et al. (2008) Safety and immunogenicity of the candidate tuberculosis vaccine MVA85A in West Africa. PLoS One 3.

22. Ibanga HB, Brookes RH, Hill PC, Owiafe PK, Fletcher HA, et al. (2006) Early clinical trials with a new tuberculosis vaccine, MVA85A, in tuberculosis-endemic countries: issues in study design. Lancet Infect Dis 6: 522-528.

23. Tameris MD, Hatherill M, Landry BS, Scriba TJ, Snowden MA, et al. (2013) Safety and efficacy of MVA85A, a new tuberculosis vaccine, in infants previously vaccinated with BCG: a randomised, placebo-controlled phase $2 \mathrm{~b}$ trial. Lancet.

24. Dye C, Fine PE (2013) A major event for new tuberculosis vaccines. Lancet.

25. Beveridge NE, Price DA, Casazza JP, Pathan AA, Sander CR, et al. (2007) Immunisation with BCG and recombinant MVA85A induces long-lasting, polyfunctional Mycobacterium tuberculosis-specific CD4+ memory T lymphocyte populations. Eur J Immunol 37: 3089-3100.

26. Minassian AM, Rowland R, Beveridge NER, Poulton ID, Satti I, et al. (2011) A Phase I study evaluating the safety and immunogenicity of MVA85A, a candidate TB vaccine, in HIV-infected adults. BMJ Open 1.

27. Scriba TJ, Tameris M, Smit E, van der Merwe L, Hughes EJ, et al. (2012) A phase IIa trial of the new tuberculosis vaccine, MVA85A, in HIV- and/or Mycobacterium tuberculosis-infected adults. Am J Respir Crit Care Med 185: 769-778.

28. Pathan AA, Sander CR, Fletcher HA, Poulton I, Alder NC, et al. (2007) Boosting BCG with recombinant modified vaccinia ankara expressing antigen 85A: different boosting intervals and implications for efficacy trials. PLoS ONE 2: e1052.

29. Ota MOC, Odutola AA, Owiafe PK, Donkor S, Owolabi OA, et al. (2011) Immunogenicity of the tuberculosis vaccine MVA85A is reduced by coadministration with EPI vaccines in a randomized controlled trial in Gambian infants. Sci Transl Med 3: 88-88.

30. Plana M, Garcia F, Darwich L, Romeu J, Lopez A, et al. (2011) The reconstitution of the thymus in immunosuppressed individuals restores CD4specific cellular and humoral immune responses. Immunology 133: 318-328.

31. Sereti I, Anthony KB, Martinez-Wilson H, Lempicki R, Adelsberger J, et al. (2004) IL-2-induced CD4+ T-cell expansion in HIV-infected patients is associated with long-term decreases in T-cell proliferation. Blood 104: 775-780.

32. Douek DC (2002) The contribution of the thymus to immune reconstitution after hematopoietic stem-cell transplantation. Cytotherapy 4: 425-426.

33. Shearer GM, Clerici M (1991) Early T-helper cell defects in HIV infection. AIDS 5: 245-253.

34. Sander CR, Pathan AA, Beveridge NE, Poulton I, Minassian A, et al. (2009) Safety and immunogenicity of a new tuberculosis vaccine, MVA85A, in Mycobacterium tuberculosis-infected individuals. Am J Respir Crit Care Med 179: 724-733.

35. Badri M, Wilson D, Wood R (2002) Effect of highly active antiretroviral therapy on incidence of tuberculosis in South Africa: a cohort study. Lancet 359: 2059 2064.

36. Notermans DW, Pakker NG, Hamann D, Foudraine NA, Kauffmann RH, et al. (1999) Immune reconstitution after 2 years of successful potent antiretroviral therapy in previously untreated human immunodeficiency virus type 1 -infected adults. J Infect Dis 180: 1050-1056.

37. Schacker TW, Bosch RJ, Bennett K, Pollard R, Robbins GK, et al. (2010) Measurement of naive CD4 cells reliably predicts potential for immune reconstitution in HIV.J Acquir Immune Defic Syndr 54: 59-62.

38. Goldsack L, Kirman JR (2007) Half-truths and selective memory: Interferon gamma, CD4(+) $\mathrm{T}$ cells and protective memory against tuberculosis. Tuberculosis (Edinb) 87: 465-473.

39. Cowley SC, Elkins KL (2003) CD4+ T cells mediate IFN-gamma-independent control of Mycobacterium tuberculosis infection both in vitro and in vivo. J Immunol 171: 4689-4699.

40. Wu CY, Kirman JR, Rotte MJ, Davey DF, Perfetto SP, et al. (2002) Distinct lineages of $\mathrm{T}(\mathrm{H}) 1$ cells have differential capacities for memory cell generation in vivo. Nat Immunol 3: 852-858.

41. Wallis RS, Vinhas SA, Johnson JL, Ribeiro FC, Palaci M, et al. (2003) Whole blood bactericidal activity during treatment of pulmonary tuberculosis. J Infect Dis 187: $270-278$. 